\title{
Neural Network Based Approach for Automotive Brake Light Parameter Estimation
}

\author{
A. V. Ortega and I. N. da Silva \\ Department of Electrical Engineering, University of São Paulo, CEP 13566-590, São Carlos, SP, Brazil
}

\begin{abstract}
The advantages offered by the electronic component LED (Light Emitting Diode) have caused a quick and wide application of this device in replacement of incandescent lights. However, in its combined application, the relationship between the design variables and the desired effect or result is very complex and it becomes difficult to model by conventional techniques. This work consists of the development of a technique, through artificial neural networks, to make possible to obtain the luminous intensity values of brake lights using SMD (Surface Mounted Device) LEDs from design data. Such technique can be used to design any automotive device that uses groups of SMD LEDs. Results of industrial applications, using SMD LED, are presented to validate the proposed technique.
\end{abstract}

Keywords: Brake light, SMD LED, neural networks, intelligent systems.

\section{Introduction}

The LED device is an electronic semiconductor component that emits light. At present time, it has been used in replacement of incandescent lights because of its advantages, such as longer useful life (around 100,000 hours), larger mechanic resistance to vibrations, lesser heating, lower electric current consumption and high fidelity of emitted light color [1].

However, in designs where incandescent lights are replaced by LEDs, some of their important characteristics must be considered, such as direct current, reverse current, vision angle and luminous intensity [2].

The SMD LEDs are suitable for use in a wide variety of electronic equipment, including cordless and cellular phones, notebook computers, hand-held products, network systems, and automotive interior applications.

In automobile industry, incandescent lights have been replaced by LEDs in the brake lights, which are a third light of brakes [3]. In these brake lights are used sets of SMD LEDs usually organized in a straight line. The approval of brake light prototypes is made through measurements of luminous intensity in different angles, and the minimum value of luminous intensity for each angle is defined according to the application [4]. Several aspects related to the physical properties of luminous intensity can be found in [13].

The main difficulty found in the development of brake lights is in finding the existent relationship between the following parameters: luminous intensity $\left(I_{V}\right)$ of the SMD LED, distance between SMD LEDs $(d)$ and number of SMD LEDs ( $n$ ), with the desired effect or result, i.e., there is a complexity in making a model by conventional techniques of modeling, which are capable to identify properly the relationship between such variables. The prototype designs of brake lights have been made through trials and errors, causing increasing costs of implementation due to time spent in this stage. Moreover, the prototype approved from this system cannot represent the best relationship cost/benefit, since few variations are obtained from configurations of approved prototypes. The artificial neural networks are applied in cases like this one, where the traditional mathematic modeling becomes complex due to nonlinear characteristic of the system. These networks are able to learn from their environment and to generalize solutions, making them attractive to this type of application.

More specifically, multilayer perceptron artificial neural networks are used to estimate all values of luminous intensity required in brake light designs, which use SMD LEDs in their structures. In these cases, several brake light configurations can be simulated from the proposed approach, and those future prototype configurations that will meet the minimum values of luminous intensity required by traffic (vehicle safety standards) regulations can be identified.

\section{Overview of automotive applications using LEDs}

Modern automotive vehicles use incandescent lamps for parking, turning, and brake lights. These red and yellow lights typically employ a standard clear incandescent bulb behind a colored lens. However, incandescent bulbs consume a disproportionately large amount of energy for the amount of colored light they project from the vehicle's lighting fixture.

Recently, automotive industries have supported the development of schemes that replace the inefficient incandescent lights described above with more efficient lights like the Light Emitting Diode. Because LEDs produce light at the wavelength necessary for automotive use, less energy is consumed by these lighting fixtures than those that use white 
light generated by incandescent bulbs. Moreover, LEDs exhibit long lifetimes that are on the order of 100,000 hours. Coupled with the ruggedness inherent in solid-state devices, this indicates that LEDs may be useful for low maintenance applications. Fast response times also make them ideal for some automotive equipment.

In [4] is demonstrated that the conversion of a turn signal from an incandescent light to LED is possible with the latest advancements in LED designs. In [1] is proposed a system based on LEDs for vehicle traffic control applications. From geometric considerations, the system requires a cluster of 200 red, amber, and green or 200 multicolor LEDs for a single three-light system. In [3] is presented a vehicle that uses LEDs in its headlights. To obtain white light from an LED, a blue LED was placed behind a phosphor that emits yellow light when stimulated by the blue. Since yellow light stimulates the red and green receptors of the eye, the resulting mix of blue and yellow light gives the appearance of white (often called "lunar white").

In [8] is described a light-emitting diode brake-light messaging (LEDBM) system that can be used to avoid rear collisions. The LEDBM is comprised of modulated LED brake lights that communicate information about a vehicle's state to any following vehicle that is equipped with an LEDBM receiver. In [11] is proposed a robust vehicle detection method that uses vision to extract bright regions brake lights. In [12] is presented a vision system dedicated to the detection of vehicles in reduced visibility conditions; this system can identify brake light luminosity in order to avoid collisions.

This paper presents an industrial application using artificial neural networks to estimate values of brake light luminous intensity from design data. Although this study is aimed at the application of LED in brake lights, the methods developed and described here can also be used in other applications, such as headlights, turn lights, rear lights, traffic lights, or any other application where SMD LEDs can be used in groups.

\section{SMD LEDs applied in brake lights}

LED is an electronic device composed by a chip of semiconductor junction that when traversed by an electric current provides a recombination of electrons and holes. Figure 1 shows the representation of a junction being polarized.

However, this recombination demands that the energy produced by free electrons can be transferred to another state. In semiconductor junctions, this energy is released in form of heat and by emission of photons, i.e., light emission [5]. In silicon and germanium the largest energy emission occurs in form of heat, with insignificant light emission. However, in other materials, such as GaAsP or GaP, the number of light photons emitted is sufficient to build a source of quite visible light [6]. This process of light emission, which is intrinsic characteristic of the LEDs, is called electroluminescence [7].
In Fig. 2 can be observed the representation of the basic structure of a SMD LED.

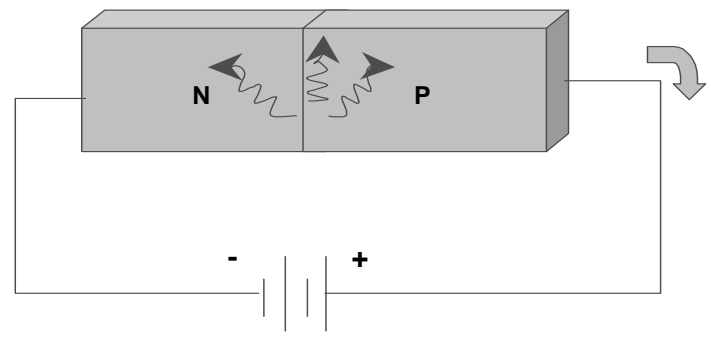

Fig. 1. Junction PN being polarized.

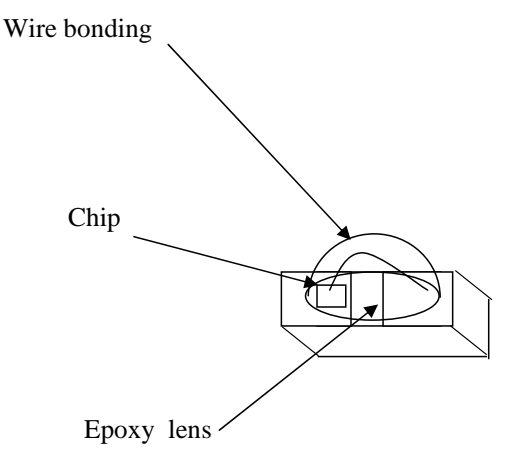

Fig. 2. Basic representation of SMD LED structure.

In brake lights the SMD LEDs are applied in set and generally organized in a straight line on a printed circuit board (PCB). In this PCB, besides the SMD LEDs, there are electronic components, basically resistors, which are responsible for the limitation of electric current that circulates through the SMD LEDs.

The main parameters used in brake lights designs are given by: SMD LED luminous intensity ( $\left.I_{V}\right)$, distance between SMD LEDs $(d)$ and number of SMD LEDs $(n)$. In Fig. 3 is illustrated a basic representation of a brake light.

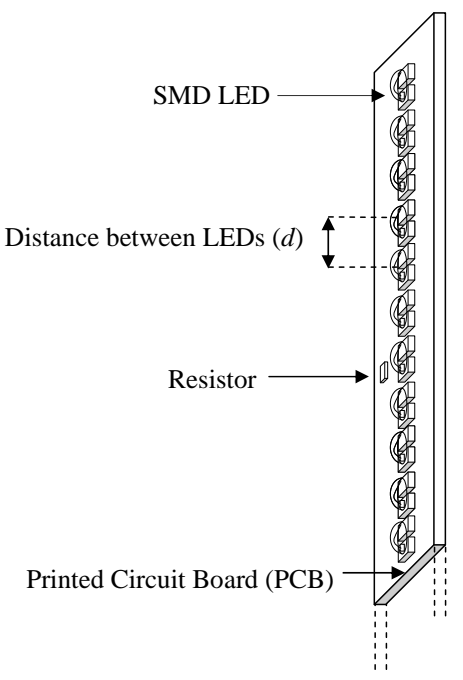

Fig. 3. Representation of a brake light. 
The main function of the brake light is to increase the safety of the vehicle (acting as a prevention system) and to reduce the risk of back collisions. Recent studies show the development of brake lights equipped with modulated signal transmitters containing information about the vehicle in which it is installed. Other vehicles that have the respective reception system of those modulated signals receive them, and their information's have been used to prevent back collisions [8]. In Fig. 4 is illustrated a brake light installed.

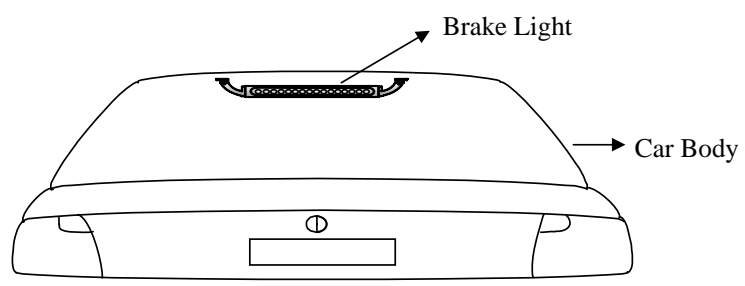

Fig. 4. Representation of a brake light installed.

At the moment there is no model or technique for designing brake lights and the prototypes are elaborated according to the common sense of designers, i.e., through trial and error methods. This occurs because the relationship between the variables involved with the light emission process of brake lights is completely nonlinear.

After elaboration of the brake light prototype, it is necessary an approval of the sample. The process for the prototype validation is made by measuring the luminous intensity of the brake light in 18 positions or different angles (Fig. 5). After this process, the values obtained in each angle are compared with those values established by governmental rules. The minimum value of luminous intensity $\left(I_{V B L}\right)$ in each angle varies according to the application. In Figure 5 is shown a representation of a generic distribution diagram of brake light luminous intensity $\left(I_{V B L}\right)$ in relation to angle. The mean horizontal position is indicated by $0^{\circ} \mathrm{H}$ and the mean vertical position is indicated by $0^{\circ} \mathrm{V}$. Thus, the position defined by the pair of angles $\left(0^{\circ} \mathrm{V}, 5^{\circ} \mathrm{L}\right)$ is represented by the shaded position shown in Fig. 5.

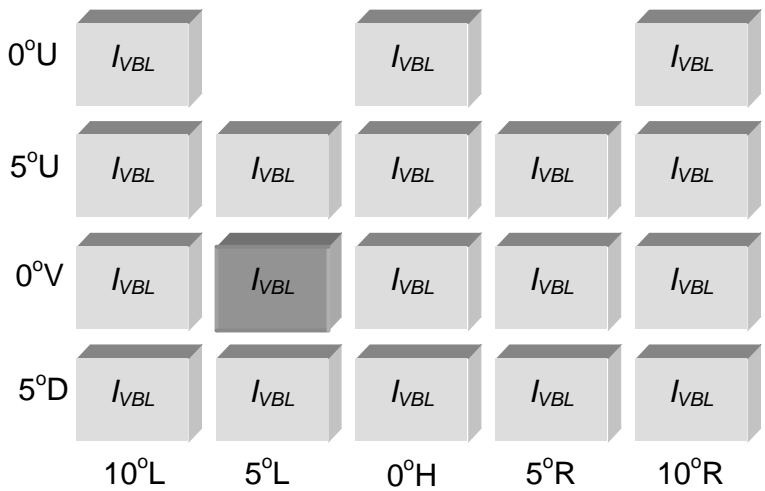

Fig. 5. Generic diagram of luminous intensity $\left(I_{V B L}\right)$ in relation to angle.

\section{Materials and methods}

For this study, 45 samples of brake lights were constructed with the following parameter variations:

- Distance between SMD LEDs ( $d$ ): 4.5 mm, 5.5 mm and $6.5 \mathrm{~mm}$.

- Number of SMD LEDs ( $n$ ): 16, 22 and 28.

- Luminous intensity of SMD LED ( $\left.I_{v}\right): 600 \mathrm{mcd}$, 800 mcd, 1200 mcd, 1500 mcd and 1800 mcd.

This combination of parameters referring to each sample can be seen in Table I. It is important to remember that the minimum and maximum values of each parameter in the designed samples must be chosen in such a way as to represent the domain for parameter variation in future designs, because these designs will be made using the proposed neural network.

A photometer was used to measure the luminous intensity of the samples, and it was coupled to a device permitting vertical and horizontal angle variation. In this way, it was possible to obtain the luminous intensity value from 18 different angles.

Table I. Combination of parameters in each sample.

\begin{tabular}{|c|c|c|c|c|c|c|c|}
\hline Sample & $\begin{array}{c}d \\
(\mathrm{~mm})\end{array}$ & $\begin{array}{c}n \\
\text { (unit) }\end{array}$ & $\begin{array}{c}I_{V} \\
\text { (mcd) }\end{array}$ & Sample & $\begin{array}{c}d \\
(\mathrm{~mm})\end{array}$ & $\begin{array}{c}n \\
\text { (unit) }\end{array}$ & $\begin{array}{c}I_{V} \\
\text { (mcd) }\end{array}$ \\
\hline 01 & 4.5 & 16 & 600 & 24 & 5.5 & 22 & 1500 \\
\hline 02 & 4.5 & 16 & 800 & 25 & 5.5 & 22 & 1800 \\
\hline 03 & 4.5 & 16 & 1200 & 26 & 5.5 & 28 & 600 \\
\hline 04 & 4.5 & 16 & 1500 & 27 & 5.5 & 28 & 800 \\
\hline 05 & 4.5 & 16 & 1800 & 28 & 5.5 & 28 & 1200 \\
\hline 06 & 4.5 & 22 & 600 & 29 & 5.5 & 28 & 1500 \\
\hline 07 & 4.5 & 22 & 800 & 30 & 5.5 & 28 & 1800 \\
\hline 08 & 4.5 & 22 & 1200 & 31 & 6.5 & 16 & 600 \\
\hline 09 & 4.5 & 22 & 1500 & 32 & 6.5 & 16 & 800 \\
\hline 10 & 4.5 & 22 & 1800 & 33 & 6.5 & 16 & 1200 \\
\hline 11 & 4.5 & 28 & 600 & 34 & 6.5 & 16 & 1500 \\
\hline 12 & 4.5 & 28 & 800 & 35 & 6.5 & 16 & 1800 \\
\hline 13 & 4.5 & 28 & 1200 & 36 & 6.5 & 22 & 600 \\
\hline 14 & 4.5 & 28 & 1500 & 37 & 6.5 & 22 & 800 \\
\hline 15 & 4.5 & 28 & 1800 & 38 & 6.5 & 22 & 1200 \\
\hline 16 & 5.5 & 16 & 600 & 39 & 6.5 & 22 & 1500 \\
\hline 17 & 5.5 & 16 & 800 & 40 & 6.5 & 22 & 1800 \\
\hline 18 & 5.5 & 16 & 1200 & 41 & 6.5 & 28 & 600 \\
\hline 19 & 5.5 & 16 & 1500 & 42 & 6.5 & 28 & 800 \\
\hline 20 & 5.5 & 16 & 1800 & 43 & 6.5 & 28 & 1200 \\
\hline 21 & 5.5 & 22 & 600 & 44 & 6.5 & 28 & 1500 \\
\hline 22 & 5.5 & 22 & 800 & 45 & 6.5 & 28 & 1800 \\
\hline 23 & 5.5 & 22 & 1200 & & & & \\
\hline
\end{tabular}

Initially, the first sample was positioned relative to a screen representing the luminous intensity diagram illustrated in Fig. 5. The photometer was placed at the first angle, and the measurement of the luminous intensity was registered. This procedure was repeated until the luminous intensity value referring to last angle of the sample was registered. Figure 6 illustrates this procedure for the pair of angles $\left(0^{\circ} \mathrm{H}\right.$, $\left.5^{\circ} \mathrm{U}\right)$ shown in Fig. 5. 


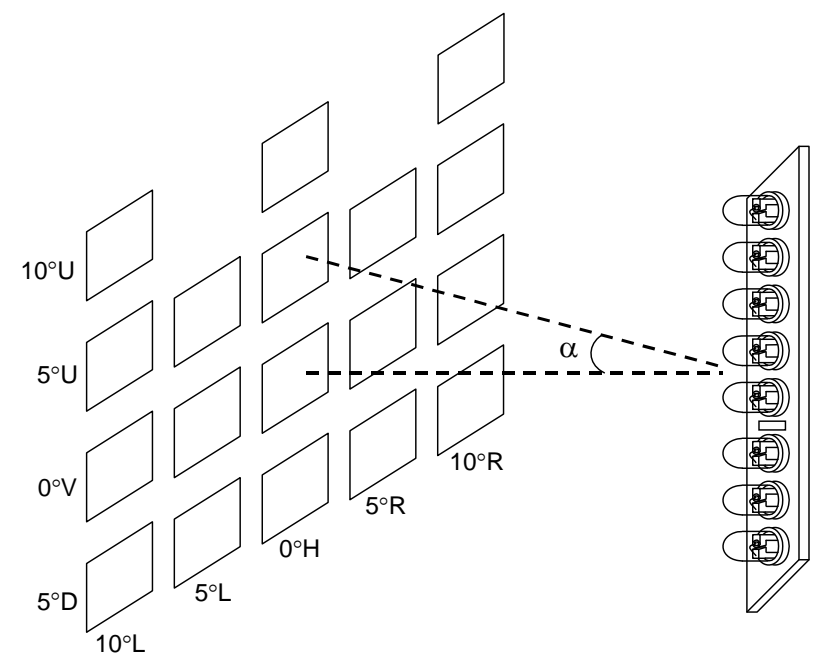

Fig 6. Luminous intensity diagram in relation to the brake light prototype.

The sample was then removed from the device, and a new sample was attached in order to measure the luminous intensity; all procedures are repeated until the value of the last angle of the last sample was registered.

From the design data provided in Table 1 and the measurement results of luminous intensity of brake light samples from different angles, a multilayer perceptron network was trained as will described. During this stage, a variation of the main network parameters was achieved. The number of layers, number of neurons per layer, activation function for each layer, and type of training were changed in order to obtain a neural network topology that could generate an acceptable mean squared error and ensure an efficient generalization. The best neural architecture for the simulations was selected by means of a cross-validation technique [10].

The topology chosen consisted of two hidden layers with 5 neurons in the first layer and 10 neurons in the second layer. The training algorithm was Levenberg-Marquardt [9]. The main advantage of this algorithm arises from its ability to accelerate the neural network convergence process, and it is considered to be the fastest method for training moderatesized perceptron networks. In comparative terms, the Levenberg-Marquardt algorithm is about 100 times faster than the backpropagation method. For our application, the network inputs were defined by the 3 main parameters involved in brake light design, i.e.

- Distance between SMD LEDs $\rightarrow d(\mathrm{~mm})$.

- Number of SMD LEDs $\rightarrow n$.

- Luminous intensity of SMD LED $\rightarrow I_{V}$ (mcd).

The network output is composed by a unique signal which provides what is the intensity level produced by the brake light in a particular angle, i.e.
- Luminous intensity of brake light $\rightarrow I_{V B L}$ (cd).

After training, using the 18 different angles, one training for each angle, it was possible to estimate the total luminous intensity produced by the brake light in different angles. To validate the proposed approach are used data coming from samples not used in the network training. A comparison between the estimated values by the network and those provided by experimental tests is accomplished to analyze the efficiency of the proposed approach.

\section{Results and discussion}

The computational implementations of the neural networks used in this application were carried out using the software Matlab/Simulink. After the training process, the neural modeling was used to obtain luminous intensity values of brake lights, as previously described. Figure 7 illustrates a comparison between luminous intensity values $\left(I_{V B L}\right)$ obtained by experimental tests (ET) and those estimated by the artificial neural network (ANN). In this configuration (Situation I), the used sample presents distance $(d)$ between SMD LEDs equal to $5.5 \mathrm{~mm}$, the number of SMD LEDs $(n)$ is equal to 28 and the luminous intensity of each SMD LED $\left(I_{v}\right)$ has a value equal to 800 mcd.

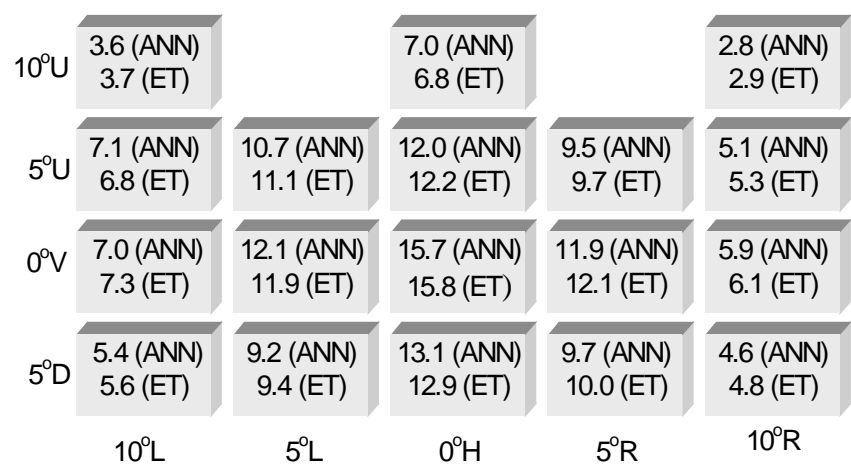

Fig. 7. Comparative illustration (Situation I).

From Fig. 7 it is observed that the generalization produced by the network to estimate values of luminous intensity in several angles is very satisfactory. In this case, the mean relative errors calculated were around $2.8 \%$ and with variance of $1.19 \%$.

Figure 8 illustrates another comparison between luminous intensity values ( $\left.I_{V B L}\right)$ obtained by experimental tests (ET) and those estimated by the artificial neural network (ANN). For this configuration (Situation II), the used sample presented the same distance $(d)$ and the same SMD LEDs number of the previous situation; but, the luminous intensity of each SMD LED ( $\left.I_{v}\right)$ has a value equal to $1200 \mathrm{mcd}$. 


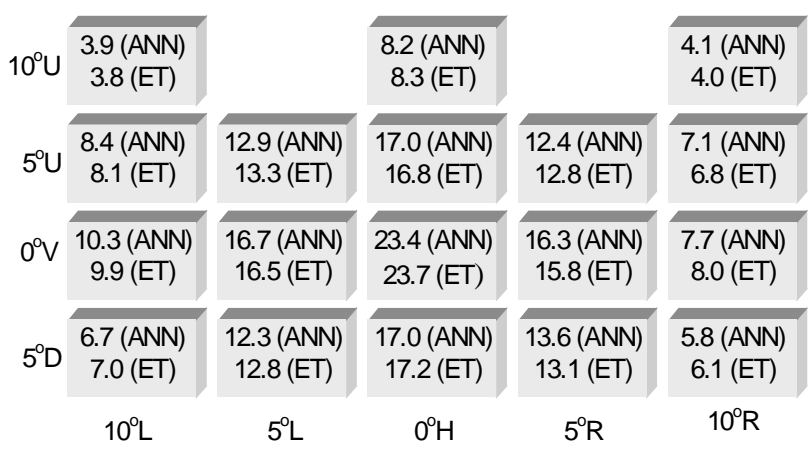

Fig. 8. Comparative illustration (Situation II).

In this case (Situation II), the mean relative errors calculated were around 3.0\% and with variance of 1.61\%.

Figure 9 illustrates another comparison between luminous intensity values ( $I_{V B L}$ ) obtained by experimental tests (ET) and those estimated by the artificial neural network (ANN). In this configuration (Situation III), the used sample presents distance $(d$ ) between SMD LEDs equal to $6.5 \mathrm{~mm}$, the number of SMD LEDs $(n)$ is equal to 22 and the luminous intensity of each SMD LED $\left(I_{v}\right)$ has a value equal to 1200 mcd.

\begin{tabular}{|c|c|c|c|c|c|}
\hline $10^{\circ} \mathrm{U}$ & $\begin{array}{c}4.5(\mathrm{ANN}) \\
4.3(\mathrm{ET})\end{array}$ & & $\begin{array}{c}7.6(\mathrm{ANN}) \\
7.4(\mathrm{ET})\end{array}$ & & $\begin{array}{c}3.1 \text { (ANN) } \\
3.0 \text { (ET) }\end{array}$ \\
\hline $5^{\circ} \mathrm{U}$ & $\begin{array}{c}7.3(\mathrm{ANN}) \\
7.6(\mathrm{ET})\end{array}$ & $\begin{array}{c}12.5 \text { (ANN) } \\
12.0(\mathrm{ET})\end{array}$ & $\begin{array}{c}14.2 \text { (ANN) } \\
14.5(\mathrm{ET})\end{array}$ & $\begin{array}{c}11.3(\mathrm{ANN}) \\
11.6(\mathrm{ET})\end{array}$ & $\begin{array}{c}6.7(\mathrm{ANN}) \\
6.9(\mathrm{ET})\end{array}$ \\
\hline $0^{\circ} \mathrm{V}$ & $\begin{array}{c}8.0(\mathrm{ANN}) \\
8.1(\mathrm{ET})\end{array}$ & $\begin{array}{c}14.2 \text { (ANN) } \\
13.8(\mathrm{ET})\end{array}$ & $\begin{array}{c}19.8 \text { (ANN) } \\
20.2 \text { (ET) }\end{array}$ & $\begin{array}{c}13.5 \text { (ANN) } \\
13.3(\mathrm{ET})\end{array}$ & $\begin{array}{c}6.9(\mathrm{ANN}) \\
7.1 \text { (ET) }\end{array}$ \\
\hline $5^{\circ} \mathrm{D}$ & $\begin{array}{c}6.1(\mathrm{ANN}) \\
6.4(\mathrm{ET})\end{array}$ & $\begin{array}{c}10.3 \text { (ANN) } \\
10.7 \text { (ET) }\end{array}$ & $\begin{array}{c}14.7 \text { (ANN) } \\
15.1 \text { (ET) }\end{array}$ & $\begin{array}{c}11.0 \text { (ANN) } \\
11.0(\text { ET) }\end{array}$ & $\begin{array}{c}6.0 \text { (ANN) } \\
5.8(\mathrm{ET})\end{array}$ \\
\hline & $10^{\circ} \mathrm{L}$ & $5^{\circ} \mathrm{L}$ & $\mathrm{O}^{\circ} \mathrm{H}$ & $5^{\circ} \mathrm{R}$ & $10^{\circ} \mathrm{R}$ \\
\hline
\end{tabular}

Fig. 9. Comparative illustration (Situation III).

In this case (Situation III), the mean relative errors calculated were around $2.9 \%$ and with variance of $1.47 \%$.

Through these results it is possible to infer that the network presented efficient results for estimation of luminous intensity values of brake lights. It should be taken into account that the proposed neural network has considered the main parameters involved with the design of brake lights. In the selection process of the best neural architecture used in simulations was adopted the cross-validation technique [10].

\section{Conclusions}

This work presents a technique based on use of artificial neural networks for determination of luminous intensity values for brake lights, in which are considered the main design characteristics. Therefore, the developed tool constitutes a new technique that can efficiently be applied in this type of problem.
The developed methodology can also be generalized and used in other applications that use groups of SMD LEDs, such as in headlights, turning lights, rear lights, traffic lights, electronic panels of messages, etc.

The developed tool has significantly contributed for reduction of costs in relation to implementation stage of brake lights, i.e, it minimizes spent time in prototype designs. The tool has also allowed simulating many options for configurations of brake lights without need of building them, which also assists in the selection process of sample that offers an appropriate relationship between cost and benefit.

\section{References}

[1] S. B. Peralta, H. E. Ruda. "Applications for advanced solid-state lamps”. IEEE Industry Applications Magazine, vol. no. 4, pp. 31-42, 1998.

[2] P. R. Edwards, R. W. Martin, I. M. Watson, C. Liu, R. A. Taylor, J. H. Rice, J. W. Robinson, J. D. Smith. "Quantum dot emission from site-controlled InGaN/GaN micropyramid arrays”. Applied Physics Letters, vol. 85, pp 4281-4283, 2004.

[3] J. Voelcher. “Top 10 tech cars”. IEEE Spectrum, vol. 41, pp. 20-27, 2004.

[4] W. R. Young, W. Wilson. "Efficient electric vehicle lighting using LEDs”. Proc. of Southcon, pp. 276-280, 1996.

[5] B. G. Streetman, S. Banerjee. Solid State Electronic Devices. Prentice Hall, Englewood Cliffs, New Jersey, 1999.

[6] R. W. Martin, P. R. Edwards, R. A. Taylor, J. H. Rice, J. W. Robinson, J. D. Smith, C. Liu, I. M. Watson. "Luminescence properties of isolated InGaN/GaN quantum dots”. Physica Status Solidi (A), vol. 202, pp. 372-376, 2005. [7] R. Pecharroman-Gallego, R. W. Martin, I. M. Watson. "Investigation of the unusual temperature dependence of InGaN/GaN quantum well photoluminescence over a range of emission energies”. Journal of Physics D: Applied Physics, vol. 21, pp. 2954-2961, 2004.

[8] P. Griffiths, D. Langer, J. A. Misener, M. Siegel, C. Thorpe. "Sensor-friendly vehicle and roadway systems". $18^{\text {th }}$ Instrumentation and Measurement Technology Conference, pp. 1036-1040, 2001.

[9] M. T. Hagan, M. B. Menhaj. "Training feedforward networks with the Marquardt algorithm”. IEEE Transactions on Neural Networks, vol. 6, pp. 989-993, 1994.

[10] S. Haykin. Neural Networks. Prentice-Hall, Upper Saddle River, New Jersey, 1999.

[11] S. Kim, S. -Y. Oh, J. Kang, Y. Ryu, K. Kim, S. -C. Park, K. Park. "Front and rear vehicle detection and tracking in the day and night times using vision and sonar sensor fusion”. IEEE/RSJ International Conference on Intelligent Robots and Systems, pp. 2173-2178, 2005.

[12] I. Cabani, G. Toulminet, A. Bensrhair. "Color-based detection of vehicle lights”. IEEE Intelligent Vehicles Symposium, pp. 278-283, 2005.

[13] P. Mottier. LED for Lighting Applications. Wiley-ISTE, Hoboken, New Jersey, 2009. 